\title{
TNF- $\alpha$ induces osteoclastogenesis by direct stimulation of macrophages exposed to permissive levels of RANK ligand
}

\author{
Jonathan Lam, ${ }^{1,2}$ Sunao Takeshita, ${ }^{1}$ Jane E. Barker, ${ }^{3}$ Osami Kanagawa, ${ }^{1}$ \\ F. Patrick Ross, ${ }^{1}$ and Steven L. Teitelbaum ${ }^{1}$ \\ ${ }^{1}$ Department of Pathology and Immunology, and \\ ${ }^{2}$ Division of Biology and Biomedical Sciences, Washington University School of Medicine, St. Louis, Missouri, USA \\ ${ }^{3}$ The Jackson Laboratory, Bar Harbor, Maine, USA \\ Address correspondence to: Steven L. Teitelbaum, Washington University School of Medicine, \\ Barnes-Jewish Hospital North, Mailstop 90-31-649, 216 S. Kingshighway Boulevard, St. Louis, Missouri 63110, USA. \\ Phone: (314) 454-8463; Fax: (314) 454-5055; E-mail: teitelbs@medicine.wustl.edu. \\ Jonathan Lam and Sunao Takeshita contributed equally to this work.
}

Received for publication August 26, 2000, and accepted in revised form November 13, 2000.

\begin{abstract}
While TNF- $\alpha$ is pivotal to the pathogenesis of inflammatory osteolysis, the means by which it recruits osteoclasts and promotes bone destruction are unknown. We find that a pure population of murine osteoclast precursors fails to undergo osteoclastogenesis when treated with TNF- $\alpha$ alone. In contrast, the cytokine dramatically stimulates differentiation in macrophages primed by less than one percent of the amount of RANKL (ligand for the receptor activator of NF- $\mathrm{KB}$ ) required to induce osteoclast formation. Mirroring their synergistic effects on osteoclast differentiation, TNF- $\alpha$ and RANKL markedly potentiate NF- $\mathrm{KB}$ and stress-activated protein kinase/c-Jun $\mathrm{NH}_{2}$-terminal kinase activity, two signaling pathways essential for osteoclastogenesis. In vivo administration of TNF- $\alpha$ prompts robust osteoclast formation in chimeric animals in which $\beta$-galactosidase positive, TNF-responsive macrophages develop within a TNF-nonresponsive stromal environment. Thus, while TNF- $\alpha$ alone does not induce osteoclastogenesis, it does so both in vitro and in vivo by directly targeting macrophages within a stromal environment that expresses permissive levels of RANKL. Given the minuscule amount of RANKL sufficient to synergize with TNF- $\alpha$ to promote osteoclastogenesis, TNF- $\alpha$ appears to be a more convenient target in arresting inflammatory osteolysis.
\end{abstract}

J. Clin. Invest. 106:1481-1488 (2000).

\section{Introduction}

The most prevalent form of clinically significant osteopenia involves chronic inflammation of bone or periosseous tissues by gram-negative bacteria, as seen in suppurative otitis media (1) and periodontitis $(2,3)$. Severe osteolysis occurring as a sequela of chronic infection reflects enhanced osteoclastic bone resorption, a process driven by host inflammatory responses $(4,5)$. Prolonged or excessive release of cytokines such as TNF- $\alpha$, IL-1, IL- 6 , and IL- 8 is central to the pathogenesis of inflammatory bone and joint destruction. A greater understanding of the molecular and cellular mechanisms by which inflammatory cytokines enhance osteoclastogenesis will provide insight into potential therapeutic and preventative strategies for inflammatory osteopenic disorders.

TNF- $\alpha$ is among the most potent of the osteoclastogenic cytokines produced in inflammation. TNF$\alpha$-induced catabolic processes are implicated in the pathogenesis of conditions including rheumatoid arthritis, orthopedic implant loosening, and other forms of chronic inflammatory osteolysis (1-5). In fact, TNF- $\alpha$ mediates lipopolysaccharide-stimulated osteoclastogenesis via its p55 receptor, through a mechanism involving activation of NF- $\mathrm{KB}(6,7)$.
Osteoclasts are bone-resorbing polykaryons derived from macrophage or myeloid-lineage progenitors under the influence of RANKL, a TNF superfamily cell-surface molecule expressed by marrow stromal cells and osteoblasts, and by activated $\mathrm{T}$ lymphocytes (8). Most osteoclastogenic factors, including TNF- $\alpha$, act via receptors on stromal or osteoblastic cells to enhance RANKL expression $(9,10)$. In contrast, several recent studies posit that TNF- $\alpha$ directly stimulates macrophages to differentiate into osteoclasts by a mechanism independent of RANKL $(11,12)$. Thus, the issue of the precise target cell(s) for the TNF- $\alpha$ effect and the nature of the role of TNF- $\alpha$ in basal and inflammatory osteoclastogenesis remain controversial.

We have examined the impact of TNF- $\alpha$ on osteoclast ontogeny. We find that TNF- $\alpha$ alone, at any concentration, fails to induce the differentiation of murine osteoclast precursors. Rather, TNF- $\alpha$ dramatically enhances in vitro osteoclastogenesis primed by a level of RANKL that is insufficient to induce osteoclast formation. Most importantly, TNF- $\alpha$ stimulates osteoclastogenesis in circumstances both in vitro and in vivo in which osteoclast precursors, but not RANKL-producing stromal cells, are responsive to the cytokine. Thus, TNF- $\alpha$ targets both marrow stromal cells and osteoclast pre- 
cursors, but directly impacts the latter only in the presence of permissive levels of RANKL.

\section{Methods}

Reagents. Recombinant murine TNF- $\alpha$, murine M-CSF, murine osteoprotegerin-human Fc chimera (OPG-Fc), and anti-murine RANK polyclonal antibody were procured from R\&D Systems Inc. (Minneapolis, Minnesota, USA). Recombinant murine RANKL was prepared as a fusion protein with glutathione $\mathrm{S}$-transferase, as we have previously described (13), and lack of endotoxin contamination was confirmed by limulus amoebocyte lysate assay (BioWhittaker Inc., Walkersville, Maryland, USA). Microbeads for immunopurification were obtained from Miltenyi Biotec (Auburn, California, USA). Anti-murine CD3, CD11b, and CD106 mAb's were purchased from PharMingen (San Diego, California, USA). Anti-murine c-Fms mAb (AFS98) was prepared by affinity chromatography from a hybridoma culture provided by Shin-Ichi Nishikawa (Kyoto University, Kyoto, Japan) (14). Except where stated, all miscellaneous reagents were obtained from Sigma Chemical Co. (St. Louis, Missouri, USA).

Mice. Four- to six-week-old male $\mathrm{C} 3 \mathrm{H} / \mathrm{HeN}$ inbred mice (Harlan Sprague Dawley Inc., Indianapolis, Indiana, USA) were employed, along with the following age-matched male transgenic animals from The Jackson Laboratory: mice deficient in both p55 and p75 TNF receptors (B6,129S-TNFrsfla-TNFrsflb) and their heterozygous littermates; and B6,129S-Gtrosa26 (Rosa) mice expressing a lac $Z$ transgene. Animals were allowed food (Prolab RMH 3000; PMI Nutrition International, St. Louis, Missouri, USA) and water ad libitum, and maintained under conditions of a 12-hour light and dark cycle.

Cell culture. Pure populations of murine myeloid or stromal/osteoblastic cells were isolated from whole bone marrow extracted from femora, tibiae, and humeri by immunopurification with antibodies directed against c-Fms, CD11b, RANK, F4/80, or CD106. Myeloid cells were cultured in $\alpha$-MEM containing $10 \%$ FBS, with stromal/osteoblastic cells receiving an additional $10 \%$ donor horse serum. Cells were incubated at $37^{\circ} \mathrm{C}$ in a humidified atmosphere containing $6 \% \mathrm{CO}_{2}$ and supplemented with fresh media and cytokines daily. Typical mature osteoclast formation was observed between culture days 5 and 9 .

Bone marrow transplantation. Age-matched ( $21 \pm 1$ day) recipient TNFR-deficient and TNFR-heterozygous animals were subjected to marrow-ablative $\gamma$-irradiation generated by a ${ }^{137} \mathrm{Cs}$ source at a rate of $0.155 \mathrm{~Gy} / \mathrm{min}$. Stromal-cell and T-cell depletion of donor Rosa marrow was performed by immunodepletion with antiCD106 and anti-CD3 antibodies. $4.2 \times 10^{7}$ stromal- and $\mathrm{T}$ cell-depleted marrow cells were administered to each irradiated recipient by intravenous injection, and engraftment was allowed to proceed for 4 weeks. In vivo T-lymphocyte depletion was performed by intraperitoneal administration of anti-CD4 and anti-CD8 ascites, as delineated elsewhere (15). Donor marrow repopulation of irradiated hosts and marrow cell phenotypes were assessed by flow cytometry.

Flow cytometry. Cells were labeled with antibodies at the manufacturers' recommended dilutions and conditions. Nonspecific signal was calculated and attenuated by incubation with fluorochrome-conjugated secondary antibody in the absence of primary antibody. Labeled cells were analyzed with a FACSCalibur flow cytometry system (Becton Dickinson Immunocytometry Systems, San Jose, California, USA).

Histochemistry. Tartrate-resistant acid phosphatase (TRAP) activity was identified with a commercially available kit (Sigma Chemical Co.). A quantitative TRAP assay was performed by addition of a colorimetric substrate, $5.5 \mathrm{mM}$ p-nitrophenyl phosphate, in the presence of $10 \mathrm{mM}$ sodium tartrate at $\mathrm{pH} 4.5$. The reaction product was quantified by measuring optical absorbance at $405 \mathrm{~nm}$. $\beta$-galactosidase immunohistochemistry was performed with a monoclonal primary antibody (Sigma Chemical Co.) and a VectaStain ABC detection system (Vector Laboratories, Burlingame, California, USA).

$R T-P C R$. RNA was isolated with the RNeasy Total RNA System (QIAGEN Inc., Valencia, California, USA), digested with deoxyribonuclease to eliminate genomic DNA, and characterized with the Platinum Quantitative RT-PCR Thermoscript System (Life Technologies Inc., Rockville, Maryland, USA). Briefly, 50 pg RNA was reverse transcribed to cDNA using murine gene-specific oligonucleotide primers designed to span exon-intron boundaries: RANKL sense GGTCGGGCAATTCTGAATT and antisense GGGAATTACAAAGTGCACCAG; $\beta$-actin sense ATGGATGACGATATCGCTG and antisense ATGAGGTAGTCTGTCAGGT; GAPDH sense ACTTTGTCAAGCTCATTTCC and antisense TGCAGCGAACTTTATTGATG; and HPRT sense GTTGGATACAGGCCAGACTTTGTTG and antisense GAGGGTAGGCTGGCCTATAGGCT. Reverse transcription was performed at $60^{\circ} \mathrm{C}$ for 30 minutes, followed by predenaturation at $95^{\circ} \mathrm{C}$ for 5 minutes. Touchdown PCR amplification immediately ensued by thermal cycling as follows: ten successive cycles of denaturation at $95^{\circ} \mathrm{C}$ for 15 seconds, annealing at $65^{\circ} \mathrm{C}$ for 1 minute, and polymerization at $72^{\circ} \mathrm{C}$ for 1 minute. During subsequent cycles, the annealing temperature was decreased stepwise by $5^{\circ} \mathrm{C}$ every five cycles. After 25 rounds of amplification, reaction products were fractionated electrophoretically in $2 \%$ agarose.

Stress-activated protein kinase/c-Jun $\mathrm{NH}_{2}$-terminal kinase activity assay. Cells were treated with $1 \mathrm{ng} / \mathrm{ml}$ RANKL and $500 \mathrm{pg} / \mathrm{ml} \mathrm{TNF}-\alpha$ for 12 minutes and triturated into hypotonic lysis solution. Substrate pulldown of stress-activated protein kinase/c-Jun $\mathrm{NH}_{2}$-terminal kinase (SAPK/JNK) and in vitro kinase assays were performed using a kit from Cell Signaling Technology (Beverly, Massachusetts, USA). Samples were fractionated by SDS-PAGE, with subsequent Western analysis according to the manufacturer's established protocol.

Electrophoretic mobility shift assay. Cells were treated with $10 \mathrm{ng} / \mathrm{ml} \mathrm{RANKL}$ and/or $1 \mathrm{ng} / \mathrm{ml} \mathrm{TNF}-\alpha$ for 15 


\section{Figure 1}

Purified myeloid cells express macrophage lineage cell surface markers and differentiate into osteoclasts when cultured with RANKL. (a) Marrow cells were isolated by immunoselection and analyzed by flow cytometry. All purified cells are negative for CD106, a stromal cell marker, and positive for the macrophage lineage markers $F 4 / 80$, cFms, and CD11b. Furthermore, the purified cells express RANK, a cellsurface receptor which is necessary for osteoclast differentiation. Cells were cultured with (b) $10 \mathrm{ng} / \mathrm{ml} \mathrm{M-CSF}$ alone, or in combination with: (c) $100 \mathrm{ng} / \mathrm{ml} \mathrm{RANKL;} \mathrm{(d)} 500 \mathrm{ng} / \mathrm{ml}$ OPG; and (e) $100 \mathrm{ng} / \mathrm{ml} \mathrm{RANKL}$ and $500 \mathrm{ng} / \mathrm{ml}$ OPG. Cells treated with RANKL differentiate into a confluent layer of multinucleated osteoclasts (panel c), a process completely inhibited by concomitant treatment with OPG (panel e).

minutes. Nuclei were isolated by standard methodology, and protein content was quantitated by BCA Protein Assay (Pierce Chemical Co., Rockford, Illinois, USA). Nuclear extracts were incubated with a radiolabeled oligonucleotide probe derived from the $\mathrm{KB} 3$ site of the TNF- $\alpha$ promoter. Samples were fractionated by PAGE through a 4-20\% gradient (Novex, San Diego, California, USA) and visualized radiographically.

\section{Results}

TNF- $\alpha$, like most osteoclastogenic agents, exerts its effect via receptors on stromal or osteoblastic cells. Hence, determining if the cytokine also directly promotes the differentiation of osteoclast precursors requires a system in which these cells exist in an environment devoid of stromal/osteoblastic contamination. To this end, a myeloid/macrophage cell population was isolated by immunoselection immediately following marrow evacuation from long bones. Using this approach, greater than $99.99 \%$ of the isolated cells fail to express the stromal/osteoblastic markers CD106 (VCAM-1) and alkaline phosphatase, but demonstrate the macrophage lineage markers RANK, F4/80, c-Fms, and CD11b (Mac1) (Figure 1a). Moreover, the population differentiates into functionally mature osteoclasts, when cultured with M-CSF and RANKL (Figure 1, b and c). In the absence of M-CSF, no viable cells remain within 3 days, and concomitant treatment with OPG, a decoy receptor for RANKL, abrogates osteoclast differentiation (Figure 1, d and e). As such, these cells are deemed to be a population of primary osteoclast precursors that is free of stromal/osteoblastic contamination. We then tested the osteoclastogenic potential of this pure population of cells under the influence of TNF- $\alpha$ alone. We find that a broad range of the cytokine, in the presence of M-CSF, fails to induce these cells to undergo osteoclast differentiation (Figure 2).

These observations stand in contrast to those of Kobayashi et al. (11) and Azuma et al. (12), who propose that TNF- $\alpha$ treatment of bone marrow macrophages induces them to acquire the osteoclast phenotype. To address this discrepancy, we utilized their approach of 3 days of whole marrow culture prior to macrophage isolation. In this circumstance we, too, observed that TNF- $\alpha$ directly induces osteoclast formation. However, a
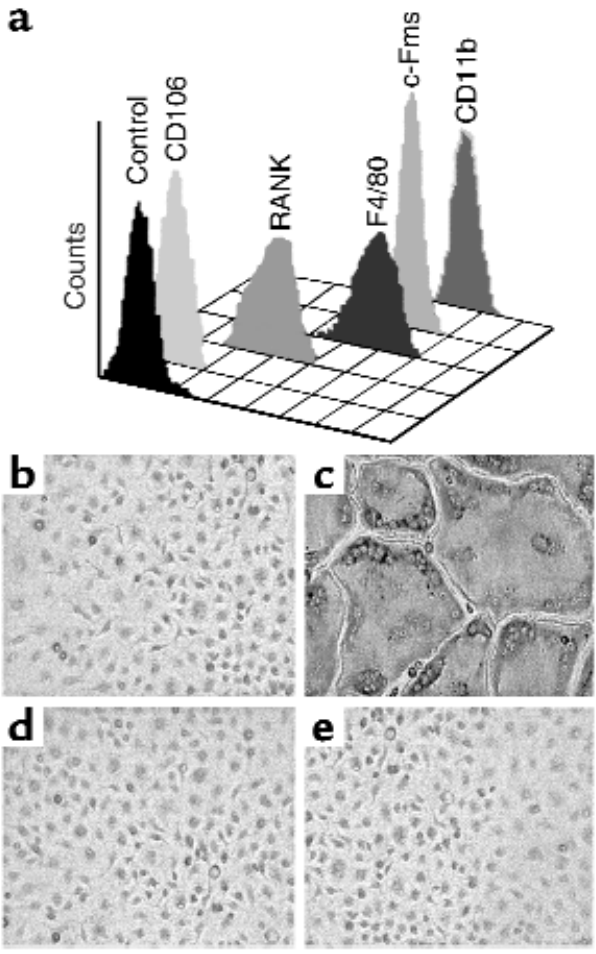

when saturating levels of OPG are added at the initiation of whole bone marrow culture, rather than concomitant with addition of TNF- $\alpha$ after the initial 3-day culture period, subsequent TNF- $\alpha$-stimulated osteoclastogenesis is completely abrogated (Figure 3 ). Similarly, when we alter our method of cell preparation to allow an overnight incubation of whole marrow cells before immunoselection, TNF- $\alpha$ is capable of inducing the formation of TRAP-positive osteoclast-like cells, a phenomenon that is obviated by adding saturating levels of OPG to the overnight culture. Given the capacity of OPG to block RANKL-induced osteoclastogenesis, these observations indicate that TNF- $\alpha$ can induce osteoclast differentiation only in precursors simultaneously or previously exposed to RANKL.

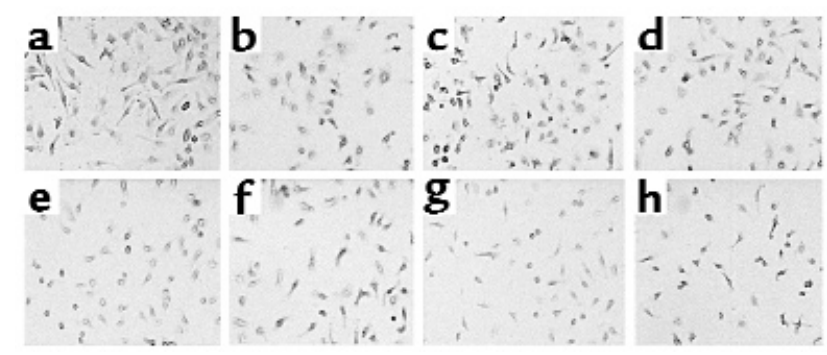

\section{Figure 2}

Purified myeloid cells fail to differentiate into osteoclasts in response to TNF- $\alpha$. Cells, as characterized in Figure 1, were cultured with 10 $\mathrm{ng} / \mathrm{ml} \mathrm{M-CSF} \mathrm{plus} \mathrm{(a)} 1 \mathrm{ng} / \mathrm{ml}$, (b) $2 \mathrm{ng} / \mathrm{ml}$, (c) $5 \mathrm{ng} / \mathrm{ml}$, (d) 10 $\mathrm{ng} / \mathrm{ml}$, (e) $20 \mathrm{ng} / \mathrm{ml}$, (f) $40 \mathrm{ng} / \mathrm{ml}$, (g) $80 \mathrm{ng} / \mathrm{ml}$, and (h) $160 \mathrm{ng} / \mathrm{ml}$ TNF- $\alpha$. Cells were stained for TRAP activity after 7 days. No multinucleated or TRAP-expressing cells are evident. 


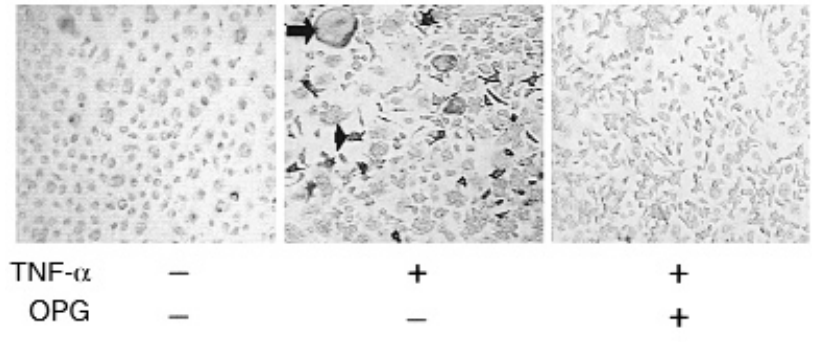

Figure 3

TNF- $\alpha$-induced differentiation of macrophages into osteoclasts is RANKL-dependent. Whole marrow was cultured with $100 \mathrm{ng} / \mathrm{ml} \mathrm{M}$ CSF, according to the method of Kobayashi et al. (11) and Azuma et al. (12) in the continuous presence or absence of $500 \mathrm{ng} / \mathrm{ml}$ OPG. After 3 days, $100 \mathrm{ng} / \mathrm{ml}$ TNF- $\alpha$ was added to some cultures. TNF- $\alpha$ induces formation of multinuclear (arrow) and mononuclear (arrowhead) TRAP-expressing cells only in the absence of OPG.

To further explore this issue, we exposed osteoclast precursors to a broad range of concentrations of both TNF- $\alpha$ and RANKL. Interestingly, in the presence of RANKL, TNF- $\alpha$ augments osteoclast differentiation in a dose-dependent manner (Figure 4). This potentiation of osteoclastogenesis is seen with all RANKL dosages beneath saturating levels.

We next asked if the synergy between TNF- $\alpha$ and RANKL is mirrored in the activation of two signaling pathways essential for osteoclastogenesis. Minimal doses of TNF- $\alpha$ or RANKL, alone, have little effect on SAPK/JNK activity (Figure 5a), or nuclear translocation of NF-KB (Figure $5 b$ ). The same minimal doses of TNF$\alpha$ and RANKL administered together, however, markedly potentiate both events.

At the cellular level, RANKL at $1 \mathrm{ng} / \mathrm{ml}$ and TNF- $\alpha$ at $1 \mathrm{ng} / \mathrm{ml}$ fail to induce osteoclast formation when

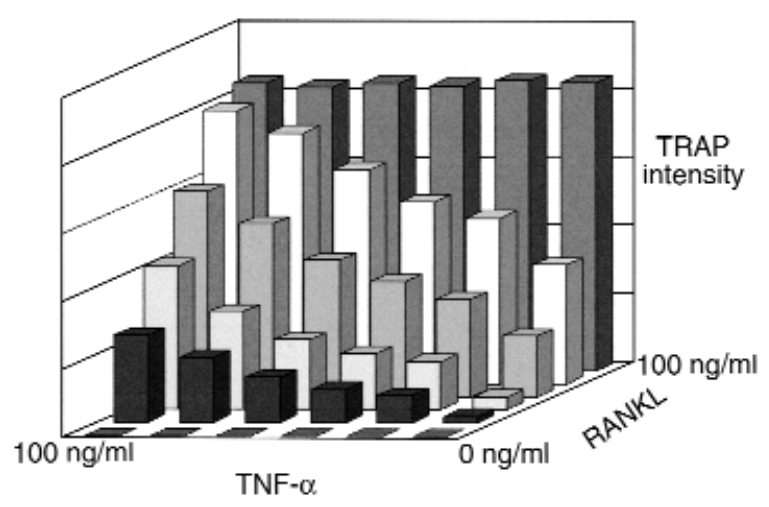

\section{Figure 4}

TNF- $\alpha$ synergizes with RANKL to stimulate osteoclast differentiation. Pure populations of myeloid cells were treated with various combinations of TNF- $\alpha$ and RANKL. After 5 days the extent of osteoclastogenesis was expressed as a function of TRAP activity, as determined by a colorimetric assay. No osteoclast formation is induced in the absence of RANKL, while in its presence, TNF- $\alpha$ augments osteoclast differentiation in a dose-dependent manner. TNF- $\alpha$ potentiation of osteoclastogenesis is seen with all RANKL dosages beneath saturating levels. administered individually. In contrast, abundant TRAP-expressing multinucleated cells appear when the cells receive this dosage of TNF- $\alpha$ following exposure to a dosage of RANKL that is less than one percent of that necessary for osteoclastogenesis. Thus, TNF- $\alpha$ is capable of potentiating subosteoclastogenic levels of RANKL (Figure 6, a-c).

The phenomenon of TNF- $\alpha$ potentiation of RANKL-primed osteoclastogenesis is time-sensitive. TNF- $\alpha$ added concomitantly with the priming dose of RANKL, immediately upon precursor purification, fails to induce osteoclast formation. In contrast, TNF$\alpha$-induced osteoclast formation is maximal when the cytokine is added $2-4$ days after RANKL priming (Figure $6 \mathrm{~d}$ ). Outside of this temporal window, TNF- $\alpha$ appears to drive macrophage development along a nonosteoclastogenic pathway.

While TNF- $\alpha$ is known to augment RANKL expression by stromal and osteoblastic cells, our data suggest that TNF- $\alpha$ may also act directly on the osteoclast precursor in the presence of basal, nonstimulated levels of RANKL. To test this hypothesis, we established cocultures of marrow macrophages with stromal cells from mice with both the p55 and p75 TNF receptors (TNFR) deleted, or from their heterozygous littermates. In this system, stromal cells from TNFR-deficient animals are capable of expressing only basal, non-TNF-inducible levels of RANKL. As seen in Figure 7a, TNF- $\alpha$ strongly stimulates osteoclast formation in these cultures in the absence of exogenous RANKL, irrespective of the ability of stromal cells to respond to TNF- $\alpha$. RT-PCR analysis (Figure $7 \mathrm{~b}$ ) confirms that the TNFR-deficient stromal cells, in coculture with TNF-responsive macrophages, fail to upregulate RANKL expression

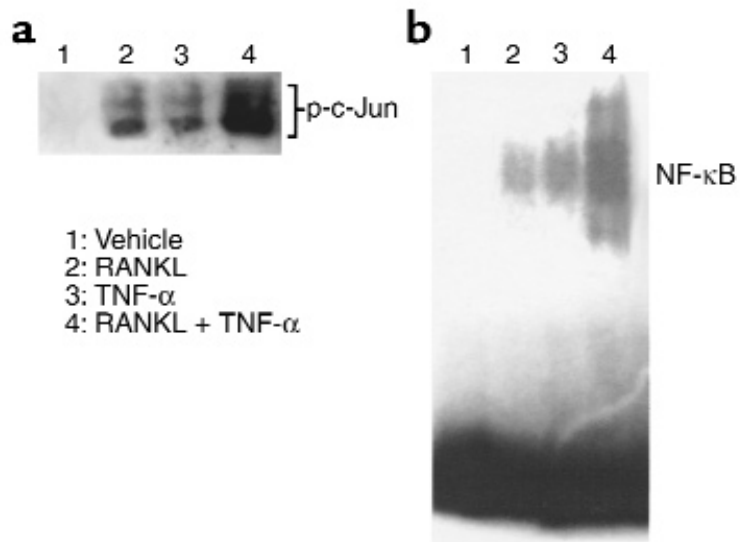

Figure 5

TNF- $\alpha$ and RANKL synergistically activate SAPK/JNK and NF- $K B$. (a) Purified myeloid cells were treated with $1 \mathrm{ng} / \mathrm{ml}$ RANKL and/or 500 $\mathrm{pg} / \mathrm{ml}$ TNF- $\alpha$ for 12 minutes. Following cell lysis, an in vitro kinase assay was performed for SAPK/JNK activity. (b) Purified myeloid cells were treated with $10 \mathrm{ng} / \mathrm{ml}$ RANKL and/or $1 \mathrm{ng} / \mathrm{ml} \mathrm{TNF-} \alpha$ for 15 minutes. Nuclear extracts were analyzed by electrophoretic mobility shift assay (EMSA), using an oligonucleotide containing the NF-KB binding site from the TNF- $\alpha$ promoter. 
over basal levels, while the TNFR-replete stromal cells do so. Thus, TNF- $\alpha$ potentiates osteoclastogenesis in vitro by directly targeting osteoclast precursors exposed to permissive levels of RANKL.

To determine if this process occurs in vivo, we took advantage of the Rosa transgenic mouse, in which $\beta$ galactosidase is constitutively expressed by all cell lineages. Stromal- and T cell-depleted marrow from Rosa mice was transplanted into irradiated TNFR-deficient and TNFR-heterozygous recipients. The resulting chimeric animals contain $\beta$-galactosidase-positive, TNF-responsive osteoclast precursors within a stromal environment that, in Rosa $\rightarrow$ TNFR-deficient animals, is not responsive to TNF- $\alpha$. In contrast, TNF- $\alpha$ is fully able to upregulate stromal cell production of RANKL in Rosa $\rightarrow$ TNFR-heterozygous animals. To eliminate the contribution of T lymphocyte-expressed RANKL, all chimeric mice were completely depleted of $\mathrm{T}$ cells in vivo. Analysis of engrafted marrow by flow cytometry confirms the absence of both $T$ lymphocytes and $\beta$ galactosidase-positive (donor) stromal cells (data not shown). The chimeric animals were administered TNF- $\alpha$ or vehicle, and osteoclast commitment was determined both ex vivo and in vivo. All generated osteoclasts are of Rosa donor origin, as demonstrated by double positivity for TRAP and $\beta$-galactosidase (Figure 8). TNF- $\alpha$, when administered in vivo, induces a marked increase in osteoclastogenesis ex vivo (Figure 9a) and in vivo (Figure 9b), within TNFR-deficient as well as TNFR-heterozygous animals. Thus, while TNF$\alpha$ alone does not stimulate osteoclastogenesis, it does so by directly targeting osteoclast precursors in the presence of a stromal environment that expresses permissive levels of RANKL.

\section{Discussion}

TNF- $\alpha$ was originally described as an endotoxininduced, macrophage-derived factor that promotes hemorrhagic necrosis of solid tumors and the cachexia of chronic infections (16). During the past decade, TNF- $\alpha$ has also been implicated in a range of inflammatory, infectious, and malignant disorders. At the cellular level, TNF- $\alpha$ modulates a broad spectrum of responses including inflammation, immunoregulation, proliferation, apoptosis, and antiviral activity (17). In bone, the cytokine inhibits extracellular matrix dep-
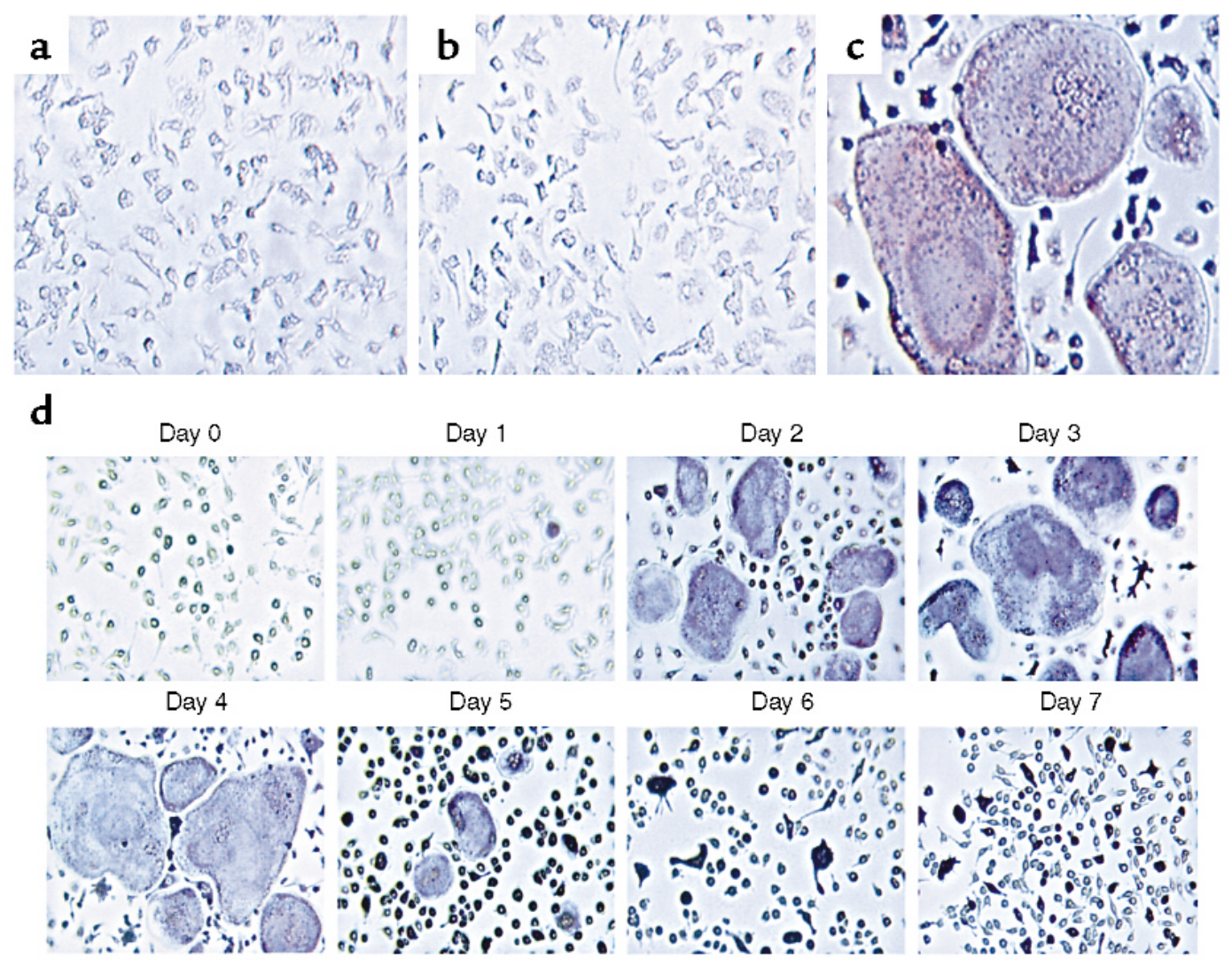

\section{Figure 6}

TNF- $\alpha$ potentiates subosteoclastogenic levels of RANKL in a time-dependent manner. Purified myeloid cells were treated with $10 \mathrm{ng} / \mathrm{ml} \mathrm{M}$ CSF plus (a) $1 \mathrm{ng} / \mathrm{ml}$ RANKL; (b) $1 \mathrm{ng} / \mathrm{ml}$ TNF- $\alpha$; and (c) $1 \mathrm{ng} / \mathrm{ml}$ RANKL plus $1 \mathrm{ng} / \mathrm{ml} \mathrm{TNF-} \alpha$. Cells were stained for TRAP activity 7 days after cytokine addition. No osteoclast formation is seen with the minimal dosage of RANKL or TNF- $\alpha$ alone, while exuberant osteoclastogenesis is evident when the cytokines act in concert. (d) Purified myeloid cells were treated with $1 \mathrm{ng} / \mathrm{ml}$ RANKL plus $10 \mathrm{ng} / \mathrm{ml}$ M-CSF. On successive days, $500 \mathrm{pg} / \mathrm{ml}$ TNF- $\alpha$ was added. Cells were stained for TRAP activity 5 days after addition of TNF- $\alpha$. TNF- $\alpha$-induced osteoclastogenesis maximizes when the cytokine is added 2-4 days following RANKL exposure. 
osition (18), stimulates matrix metalloprotease synthesis (18), and enhances production of osteoclastogenic cytokines such as M-CSF and RANKL $(19,20)$. Importantly, TNF- $\alpha$ promotes bone resorption both in vitro and in vivo by enhancing the proliferation and differentiation of osteoclast precursors (21-24).

The present study was prompted by the controversy surrounding the target cell of TNF- $\alpha$ in the osteoclastogenic process. While some studies report that TNF- $\alpha$ can stimulate osteoclast formation independent of RANKL $(11,12)$, the failure of RANK-deficient mice to mount a significant osteoclastic response in the presence of experimental inflammatory arthritis speaks against this position, as does the observation that OPG can abrogate bone erosion in this model (25). In fact, administration of TNF- $\alpha$ to RANK-defi-
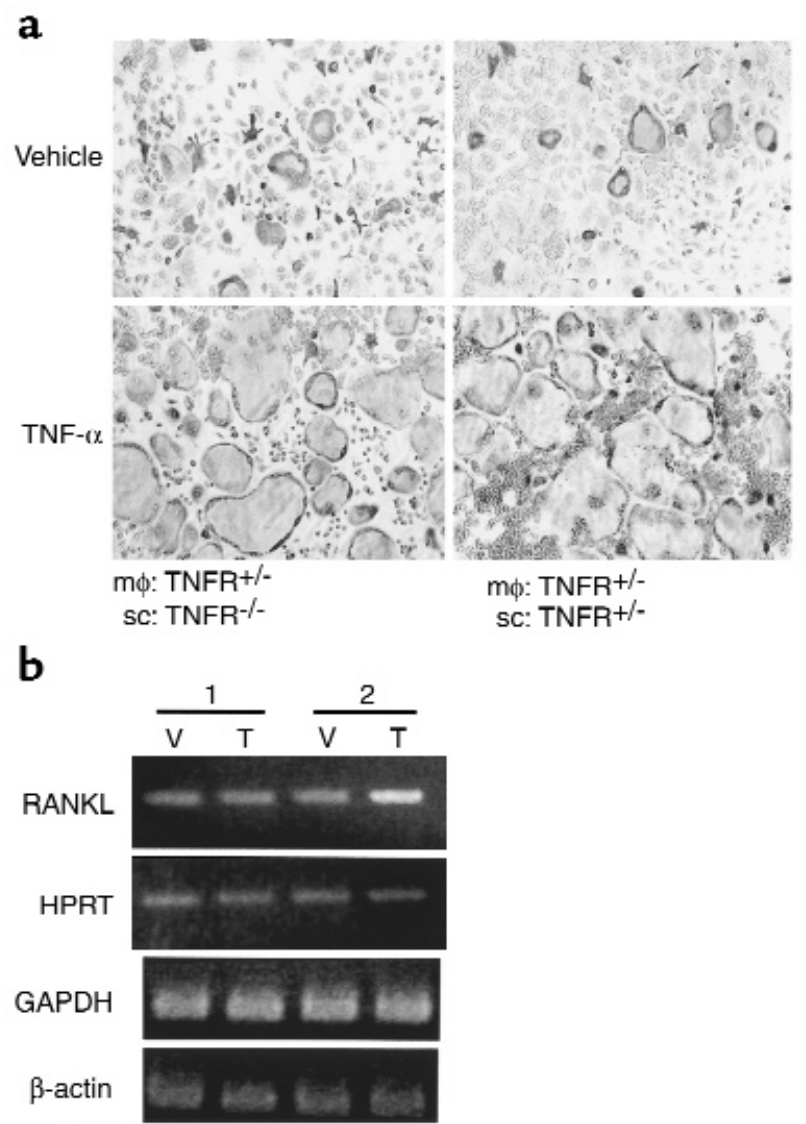

1: $\mathrm{TNFR}^{+/-} \mathrm{mo}, \mathrm{TNFR}^{-/-} \mathrm{sc}$

2: $\mathrm{TNFR}^{+/-} \mathrm{mo}, \mathrm{TNFR}^{+/-} \mathrm{SC}$

\section{Figure 7}

TNF- $\alpha$, in vitro, acts directly on osteoclast precursors to enhance osteoclastogenesis primed by basal levels of RANKL. (a) Purified TNFR-heterozygous macrophages $(\mathrm{m} \phi)$ were cultured with purified TNFR-deficient or TNFR-heterozygous stromal cells (sc), in the presence of 10 $\mathrm{ng} / \mathrm{ml} \mathrm{TNF-} \alpha$ or vehicle. Exuberant osteoclastogenesis occurs regardless of the ability of stromal cells to respond to TNF- $\alpha$. (b) RANKL expression in these cultures was analyzed by RT-PCR. Expression of RANKL is elevated by administration ofTNF- $\alpha(T)$ relative to vehicle $(V)$ only in the cultures containing TNF-responsive stromal cells. cient animals fails to induce osteoclastogenesis and ameliorate serum hypocalcemia, demonstrating that TNF- $\alpha$ does not substitute for RANKL in physiological conditions (26).

Our first exercise was to examine the proposition that TNF- $\alpha$-stimulated macrophages can differentiate into osteoclasts in the absence of RANKL. Confirmation of this posture required a pure population of osteoclast precursors not previously activated by RANKL. To this end, we not only selected macrophages by immunopurification, but also isolated them from the marrow space in the presence of OPG, a soluble decoy receptor that competes with RANK for RANKL (27). We found no amount of TNF- $\alpha$ capable of prompting osteoclast development in these purified, RANKL-deprived primary macrophages.

While these data suggested that TNF- $\alpha$ alone cannot directly induce marrow macrophages to assume the osteoclast phenotype, we substantiated the results of others who have induced osteoclast differentiation with TNF- $\alpha$ alone $(11,12)$. In contrast to our approach, their method involves maintenance of whole marrow in culture for up to 5 days prior to macrophage isolation. We reasoned that RANKL, present in these marrow cultures on the surface of stromal and osteoblastic cells, may prime macrophages to subsequently undergo osteoclast differentiation when exposed only to TNF- $\alpha$. This hypothesis was confirmed by a series of experiments. First, we documented that macrophages isolated from marrow cultures containing OPG are incapable of TNF- $\alpha$-induced osteoclastogenesis. Next, we noted that while purified macrophages fail to form osteoclasts in response to TNF- $\alpha$, overnight culture of the same marrow prior to immunopurification enables them to do so. Finally, we documented that immunopurified macrophages, recovered from bone in the presence of OPG, directly respond to TNF- $\alpha$ following pre-exposure to low levels of RANKL.

Our findings indicate that a profound synergy exists between RANKL and TNF- $\alpha$ in the osteoclastogenic process. It was therefore of interest to determine if this synergy is reflected in signaling pathways essential for osteoclast differentiation. Two critical distal events in osteoclastogenesis are activation of NF- $\kappa \mathrm{B}$ and SAPK/JNK. Mice with the p50 and p52 NF-кB subunits deleted fail to generate osteoclasts and develop osteopetrosis (28). Likewise, mice deficient in c-Fos, an integral component of the AP-1 heterodimer activated by the SAPK/JNK pathway, are also osteopetrotic (29). While low levels of either RANKL or TNF- $\alpha$ individually activate both NF- $\mathrm{KB}$ and SAPK/JNK, concomitant presence of the cytokines markedly enhances this activation, an observation that mirrors the cooperative impact of these agents on osteoclastogenesis.

To determine if TNF- $\alpha$ is capable of directly inducing macrophages to differentiate into osteoclasts by potentiating basal, non-TNF- $\alpha$-inducible levels of RANKL, 


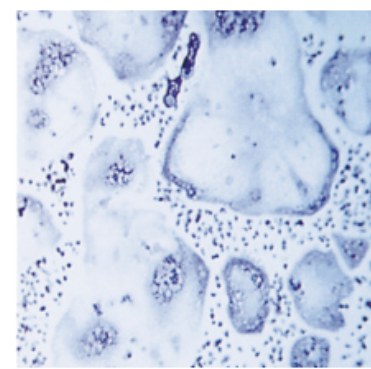

TRAP

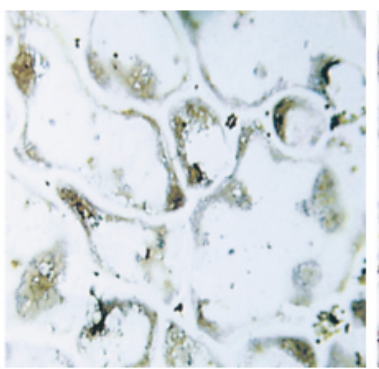

$\beta$-gal

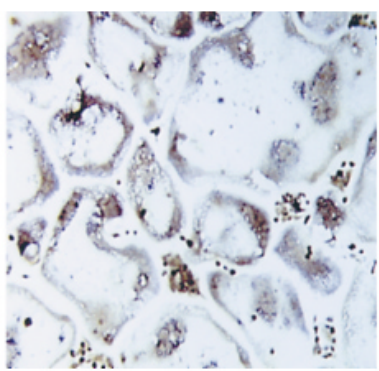

TRAP $+\beta$-gal

\section{Figure 8}

Rosa macrophages differentiate into $\beta$-galactosidase-positive, committed osteoclast precursors when transplanted into TNFR-heterozygous mice. Bone marrow transplantation was employed to create chimeric animals in which $\beta$-galactosidase-positive osteoclast precursors exist within the stromal environment of TNFR-heterozygous mice. Following engraftment, marrow cells were cultured for 7 days in osteoclastogenic conditions. Generated osteoclasts were stained for TRAP and $\beta$-galactosidase ( $\beta$-gal) activity, alone and in combination. TRAP histochemical staining yields a purple reaction product, while $\beta$-galactosidase immunohistochemical staining yields a brown reaction product. Double staining for both TRAP and $\beta$-galactosidase yields combined reaction products.

The fact that abundant osteoclasts appear in these cultures when exposed to TNF- $\alpha$ in the absence of exogenous RANKL substantiates that, at least in vitro, the cytokine induces osteoclastogenesis by targeting macrophages exposed to permissive levels of RANKL.

The fact that cells derived from Rosa transgenic mice are identified by $\beta$-galactosidase expression afforded the opportunity to confirm these observations both ex vivo and in vivo. Marrow from Rosa mice was transplanted into marrowablated recipients lacking both TNF receptors. The stromal cells in these chimeric animals are unable to recognize TNF- $\alpha$, whereas the osteoclast precur-

we employed an osteoclastogenic coculture system containing only marrow macrophages and stromal cells. This model provides an opportunity to observe osteoclast formation by TNF-responsive macrophages cultured with TNF-nonresponsive stromal cells, which can express only basal, nonstimulated levels of RANKL. sors are capable of doing so. Mirroring the in vitro situation, the numbers of committed osteoclast precursors, as determined by ex vivo culture, and the numbers of fully differentiated osteoclasts in vivo, evident in histological sections of bone, are markedly increased by systemic administration of TNF- $\alpha$ in these animals, a
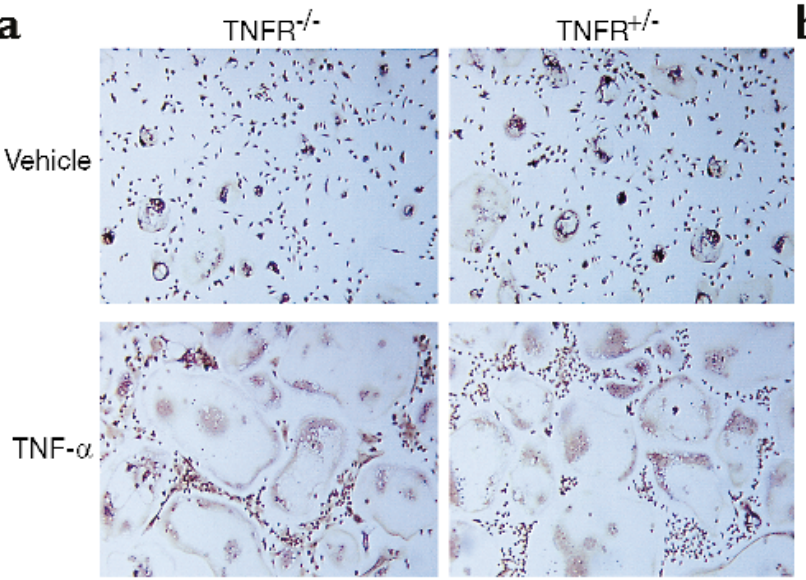

b

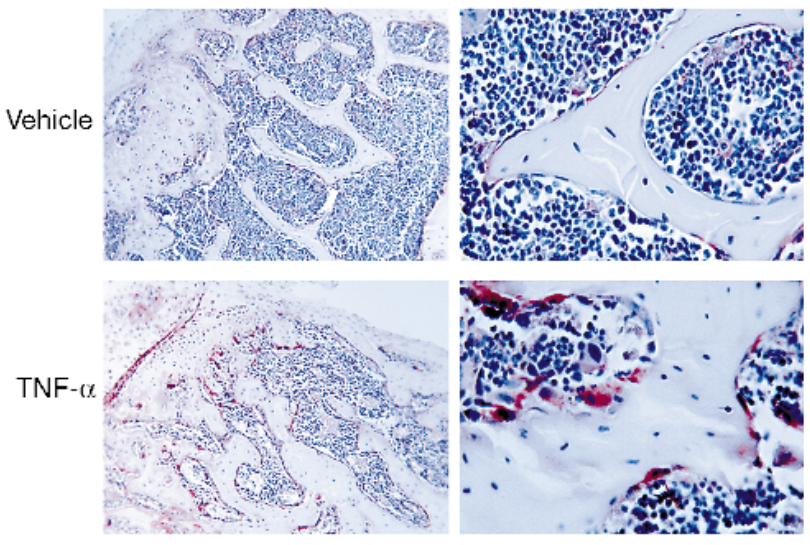

$\mathrm{TNFR}^{+/-}$

\section{Figure 9}

TNF- $\alpha$ directly induces myeloid cells to differentiate into committed osteoclast precursors and mature osteoclasts in vivo, irrespective of the capacity of stromal cells to respond to the cytokine. Chimeric animals were created by bone marrow transplantation in which $\beta$-galactosidase-positive osteoclast precursors exist within the stromal environments of TNFR-deficient animals or their TNFR-heterozygous littermates. Following engraftment, the animals were depleted of T cells and administered $150 \mu \mathrm{g} / \mathrm{kg} /$ day TNF- $\alpha$ or vehicle for 5 days by subcutaneous injection. (a) Equal numbers of whole marrow cells were then cultured ex vivo for 7 days in osteoclastogenic conditions, and stained for TRAP and $\beta$-galactosidase activity in combination. (b) Decalcified sections of long bones, representative of data from six chimeric animals, were stained for TRAP activity (red reaction product) (left panels, $\times 100$; right panels, $\times 250$ ).

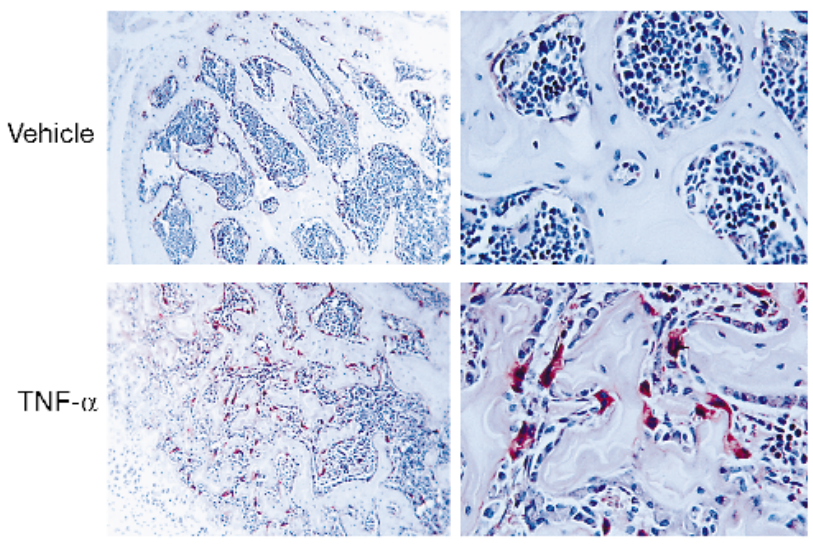


despite the inability of the stromal environment to respond to the cytokine by upregulation of RANKL.

Clearly, the osteoclastogenic relationship of TNF- $\alpha$ and RANKL is complex. Both agents, individually and in combination, greatly impact osteoclast ontogeny. The current paradigm for the role of TNF- $\alpha$ in inflammatory osteolysis holds that the cytokine acts on the stromal environment to enhance expression of osteoclastogenic factors, thereby increasing the magnitude of osteoclast differentiation. Our data suggest that TNF- $\alpha$ also acts directly on the osteoclast precursor to potentiate RANKL-induced osteoclastogenesis, even in the absence of elevated levels of RANKL. In states of inflammation, TNF- $\alpha$ concomitantly employs both pathways to enhance acquisition of the osteoclast phenotype by macrophages. Perhaps the most compelling message of this study is the fact that TNF- $\alpha$ stimulates exuberant osteoclastogenesis in vivo, even if basal levels of RANKL are not upregulated. Less than one percent of RANKL required for osteoclast differentiation in vitro is sufficient to synergize with TNF- $\alpha$ to prompt robust osteoclast development. Thus, in states of bone loss associated with excess TNF- $\alpha$, pharmacological blockade of RANKL will be successful only if this molecule is virtually eliminated. TNF- $\alpha$ may therefore be a more convenient clinical target than RANKL in arresting inflammatory osteolysis.

\section{Acknowledgments}

This study was supported by NIH grants AR07033 (J. Lam); AR32788, AR46523, and DE05413 (S.L. Teitelbaum); AR46852 (F.P. Ross); and DK27726 (J.E. Barker); and by a grant from the Shriners' Hospitals. J. Lam is the recipient of National Research Service Award grant 5T32GM07200-25, the Steven L. Teitelbaum Distinguished Alumni Scholarship for Medical Studies (jointly funded by the Washington University Medical Center Alumni Association and the Washington University School of Medicine), and an A $\Omega$ A Medical Honor Society Research Fellowship Award.

1. Lannigan, F.J., O’Higgins, P., and McPhie, P. 1993. The cellular mechanism of ossicular erosion in chronic suppurative otitis media. J. Laryngol. Otol. 107:12-16

2. Baroukh, B., and Saffar, J.L. 1991. Identification of osteoclasts and their mononuclear precursors. A comparative histological and histochemical study in hamster periodontitis. J. Periodontal Res. 26:161-166.

3. Nair, S.P., et al. 1996. Bacterially induced bone destruction: mechanisms and misconceptions. Infect. Immun. 64:2371-2380.

4. Bom-van Noorloos, A.A., et al. 1990. Bacteroides gingivalis stimulates bone resorption via interleukin-1 production by mononuclear cells. $J$. Clin. Periodontol. 17:409-413.

5. Birkedal-Hansen, H. 1993. Role of cytokines and inflammatory mediators in tissue destruction. J. Periodontal Res. 28:500-510.
6. Abu-Amer, Y., Ross, F.P., Edwards, J., and Teitelbaum, S.L. 1997. Lipopolysaccharide-stimulated osteoclastogenesis is mediated by tumor necrosis factor via its p55 receptor. J. Clin. Invest. 100:1557-1565.

7. Abu-Amer, Y., et al. 1998. Tumor necrosis factor- $\alpha$ activation of nuclear transcription factor- $\mathrm{\kappa B}$ in marrow macrophages is mediated by c-src tyrosine phosphorylation of IкB $\alpha$. J. Biol. Chem. 273:29417-29423.

8. Teitelbaum, S.L. 2000. Bone resorption by osteoclasts. Science. 289:1504-1508.

9. Quinn, J.M., Horwood, N.J., Elliott, J., Gillespie, M.T., and Martin, T.J. 2000. Fibroblastic stromal cells express receptor activator of NF- $\kappa B$ ligand and support osteoclast differentiation. J. Bone. Miner. Res. 15:1459-1466.

10. Ross, F.P. 2000. RANKing the importance of measles virus in Paget's disease. J. Clin. Invest. 105:555-558.

11. Kobayashi, K., et al. 2000. Tumor necrosis factor- $\alpha$ stimulates osteoclast differentiation by a mechanism independent of the ODF/RANKLRANK interaction. J. Exp. Med. 191:275-286.

12. Azuma, Y., Kaji, K., Katogi, R., Takeshita, S., and Kudo, A. 2000. TNF- $\alpha$ induces differentiation of and bone resorption by osteoclasts. J. Biol. Chem. 275:4858-4864

13. McHugh, K.P., et al. 2000. Mice lacking $\beta 3$ integrins are osteosclerotic because of dysfunctional osteoclasts. J. Clin. Invest. 105:433-440.

14. Sudo, T., et al. 1995. Functional hierarchy of c-kit and c-fms in intramarrow production of CFU-M. Oncogene. 11:2469-2476.

15. Coligan, J.E., Kruisbeek, A.M., Margulies, D.H., Shevach, E.M., and Strober, W. 2000. In vivo depletion of CD-4 and CD-8 specific T-cells. In Current protocols in immunology. R. Coico, series editor. John Wiley \& Sons Inc. New York, New York, USA. 4.1.

16. Beutler, B., and Cerami, A. 1987. Cachectin: a monokine implicated as a mediator of cachexia and shock. Lymphokines. 14:203-222.

17. Ledgerwood, E.C., Pober, J.S., and Bradley, J.R. 1999. Recent advances in the molecular basis of TNF signal transduction. Lab. Invest. 79:1041-1050.

18. Siwik, D.A., Chang, D.L., and Colucci, W.S. 2000. Interleukin-1 $\beta$ and tumor necrosis factor- $\alpha$ decrease collagen synthesis and increase matrix metalloproteinase activity in cardiac fibroblasts in vitro. Circ. Res. 86:1259-1265.

19. Hofbauer, L.C., et al. 1999. Interleukin- $1 \beta$ and tumor necrosis factor- $\alpha$, but not interleukin-6, stimulate osteoprotegerin ligand gene expression in human osteoblastic cells. Bone. 25:255-259.

20. Horwood, N.J., Elliott, J., Martin, T.J., and Gillespie, M.T. 1998. Osteotropic agents regulate the expression of osteoclast differentiation factor and osteoprotegerin in osteoblastic stromal cells. Endocrinology. 139:4743-4746.

21. Kitazawa, R., Kimble, R.B., Vannice, J.L., Kung, V.T., and Pacifici, R. 1994. Interleukin-1 receptor antagonist and tumor necrosis factor binding protein decrease osteoclast formation and bone resorption in ovariectomized mice. J. Clin. Invest. 94:2397-2406.

22. Thomson, B.M., Mundy, G.R., and Chambers, T.J. 1987. Tumor necrosis factors $\alpha$ and $\beta$ induce osteoblastic cells to stimulate osteoclastic bone resorption. J. Immunol. 138:775-779.

23. Lerner, U.H., and Ohlin, A. 1993. Tumor necrosis factors $\alpha$ and $\beta$ can stimulate bone resorption in cultured mouse calvariae by a prostaglandin-independent mechanism. J. Bone Miner. Res. 8:147-155.

24. van der Pluijm, G., et al. 1991. Two distinct effects of recombinant human tumor necrosis factor- $\alpha$ on osteoclast development and subsequent resorption of mineralized matrix. Endocrinology. 129:1596-1604.

25. Kong, Y.Y., et al. 1999. Activated T cells regulate bone loss and joint destruction in adjuvant arthritis through osteoprotegerin ligand. Nature. 18:304-309.

26. Li, J., et al. 2000. RANK is the intrinsic hematopoietic cell surface receptor that controls osteoclastogenesis and regulation of bone mass and calcium metabolism. Proc. Natl. Acad. Sci. USA. 97:1566-1571.

27. Simonet, W.S., et al. 1997. Osteoprotegerin: a novel secreted protein involved in the regulation of bone density. Cell. 89:309-319.

28. Iotsova, V., et al. 1997. Osteopetrosis in mice lacking NFKB1 and NFKB2. Nat. Med. 11:1285-1289.

29. Grigoriadis, A.E., et al. 1994. c-Fos: a key regulator of osteoclastmacrophage lineage determination and bone remodeling. Science. 266:443-448. 